\title{
Wrist Joint Extensor Tendons
}

National Cancer Institute

\section{Source}

National Cancer Institute. Wrist Joint Extensor Tendons. NCI Thesaurus. Code C161386.

The tendons located along the back part of the forearm that cross to the thumb side of the wrist and connect muscles of the forearm to the base of hand bones, enabling bending of the wrist back and towards the thumb. 